Notfall Rettungsmed $2013 \cdot 16: 431$

DOI 10.1007/s10049-013-1703-0

Online publiziert: 4. Oktober 2013

(c) Springer-Verlag Berlin Heidelberg 2013

\section{H.-J. Busch ${ }^{1}$ H.R. Arntz ${ }^{2}$}

${ }^{1}$ Universitäts-Notfallzentrum Freiburg, Universitätsklinikum Freiburg

2 Medizinische Klinik II, Kardiologie und Pulmonologie, Charité -

Universitätsmedizin Berlin, Campus Benjamin Franklin, Berlin

\title{
Neurologie - Seltene Notfallbilder
}

Eine moderne Notfallmedizin sollte für alle akut erkrankten Menschen die optimale medizinische Betreuung gewährleisten können. Aufgrund vieler zeitkritischer Faktoren sowie moderner Behandlungsstrategien wird die Akut- und Notfallmedizin jedoch zunehmend komplexer. Zudem kommt es durch eine zunehmende Öffentlichkeitsarbeit der einzelnen Fachgebiete zur verstärkten Aufklärung der Bevölkerung mit Inanspruchnahme der präklinischen wie auch der klinischen Notfallmedizin.

\section{D) $10-15 \%$ der Patienten in zentralen bzw. interdisziplinären Notaufnahmen zeigen neurologische Symptome}

Notaufnahmen bzw. die Notfallmedizin sind für die neurologische Versorgung der Bevölkerung von großer Bedeutung. So ergibt sich ein Anteil von 10-15\% der Patienten mit neurologischen Symptomen in zentralen bzw. interdisziplinären Notaufnahmen, aber auch im Notarztdienst. Die Erstversorgung und Ersteinschätzung von Notfallpatienten erfolgt oft durch ärztliches Personal in den Notarztdiensten oder in der Notaufnahme, also Nichtneurologen. Aus diesem Grund ist es von immenser Bedeutung, dass Ärzte in der Akut und Notfallmedizin gut geschult sind, um auch bei seltenen Erkrankungen Ressourcen richtig einteilen $\mathrm{zu}$ können oder gleich den spezifischen Behandlungsoptionen zuführen zu können.

Die Beiträge des vorliegenden Leitthemenhefts „Neurologie - Seltene Notfallbilder" beschäftigt sich mit neurologi- schen Erkrankungsbildern, die - zumindest für nichtneurologische Notfallmediziner - nicht sehr häufig in den entsprechenden Diensten vorkommen.

J. Bardutzky schreibt in seinem Beitrag „Gullian-Barré-Syndrom - Was der erstversorgende Arzt wissen sollte “ über die spezifischen Symptome, Differenzialdiagnosen und die erweiterten Behandlungsoptionen des Gullian-Barré-Syndroms. Gerade hier sind zeitkritisch initiale wichtige Entscheidungen zu treffen, sodass der Patient optimal in einer neurologischen Behandlungseinheit therapiert werden kann.

R. Kollmar spricht in seinem Artikel „Transistorische globale Amnesie (TGA) - Relevanz im Notarztdienst und in der Notaufnahme" die transiente globale Amnesie (TGA) als akut einsetzende, benigne Gedächtnisstörung an, die von anderen potenziell lebendsbedrohlichen zerebralen Ereignissen abgegrenzt werden muss. Wenn die Diagnose gestellt ist, können wichtige Ressourcen geschont und die betroffenen Patienten über die Gutartigkeit der Erkrankung aufgeklärt werden.

Mit herzlichen Grüßen

Hans-Jörg Busch

Hans-Richard Arntz

\section{Korrespondenzadresse}

PD Dr. H.-J. Busch

Universitäts-Notfallzentrum Freiburg, Universitätsklinikum Freiburg Sir-A.-Krebs-Straße, 79106 Freiburg hans-joerg.busch@uniklinik-freiburg.de

\section{Einhaltung der ethischen Richtlinien}

Interessenkonflikt. H.-J. Busch und H.-R. Arntz geben an, dass kein Interessenkonflikt besteht.

Dieser Beitrag beinhaltet keine Studien an Menschen oder Tieren. 